\title{
Vascular coupling induces synchronization, quasiperiodicity, and chaos in a nephron tree
}

Marsh, D. J.; Sosnovtseva, Olga; Mosekilde, Erik; Holstein-Rathlou, Niels-Henrik

\section{Published in:}

Chaos

Link to article, DOI:

$10.1063 / 1.2404774$

Publication date:

2007

Document Version

Publisher's PDF, also known as Version of record

Link back to DTU Orbit

Citation (APA):

Marsh, D. J., Sosnovtseva, O., Mosekilde, E., \& Holstein-Rathlou, N-H. (2007). Vascular coupling induces synchronization, quasiperiodicity, and chaos in a nephron tree. Chaos, 17(1), 015114. https://doi.org/10.1063/1.2404774

\section{General rights}

Copyright and moral rights for the publications made accessible in the public portal are retained by the authors and/or other copyright owners and it is a condition of accessing publications that users recognise and abide by the legal requirements associated with these rights.

- Users may download and print one copy of any publication from the public portal for the purpose of private study or research.

- You may not further distribute the material or use it for any profit-making activity or commercial gain

- You may freely distribute the URL identifying the publication in the public portal 


\title{
Vascular coupling induces synchronization, quasiperiodicity, and chaos in a nephron tree
}

\author{
Donald J. Marsh ${ }^{a)}$ \\ Department of Molecular Pharmacology, Physiology, and Biotechnology, Brown University, \\ Providence, Rhode Island 02912 \\ Olga V. Sosnovtseva and Erik Mosekilde \\ Department of Physics, Danish Technical University, 2800 Kgs. Lyngby, Denmark \\ Niels-Henrik Holstein-Rathlou \\ Department of Medical Physiology, Panum Institute, University of Copenhagen, \\ 2200 Copenhagen N, Denmark
}

(Received 4 August 2006; accepted 10 November 2006; published online 30 March 2007)

\begin{abstract}
The paper presents a study of synchronization phenomena in a system of 22 nephrons supplied with blood from a common cortical radial artery. The nephrons are assumed to interact via hemodynamic and vascularly propagated coupling, both mediated by vascular connections. Using anatomic and physiological criteria, the nephrons are divided into groups: cortical nephrons and medullary nephrons with short, intermediate and long Henle loops. Within each of these groups the delay parameters of the internal feedback regulation are given a random component to represent the internephron variability. For parameters that generate simple limit cycle dynamics in the pressure and flow regulation of single nephrons, the ensemble of coupled nephrons showed steady state, quasiperiodic or chaotic dynamics, depending on the interaction strengths and the arterial blood pressure. When the solutions were either quasiperiodic or chaotic, cortical nephrons synchronized to a single frequency, but the longer medullary nephrons formed two clusters with different frequencies. Under no physiologically realistic combination of parameters did all nephrons assume a common frequency. Our results suggest a greater variability in the nephron dynamics than is apparent from measurements performed on cortical nephrons only. This variability may explain the development of chaotic dynamics in tubular pressure records from hypertensive rats. (C) 2007 American Institute of Physics.
\end{abstract}

[DOI: 10.1063/1.2404774]

The kidneys provide important long-term regulation of the cardiovascular system by maintaining the volume and composition of the body fluids within narrow bounds. Maintenance of the blood volume, for example, ensures that the heart can provide cardiac output appropriate to the functional needs of the organism and can maintain the arterial blood pressure at levels that permit adequate perfusion of all organs. At the same time, the kidneys protect their own function against short-term variations in the blood pressure. This regulation takes place mainly through tubuloglomerular feedback, a mechanism in which the individual nephrons of the kidney can regulate their incoming blood flow in response to variations in the composition of tubular fluid after flow dependent epithelial transport mechanisms have altered concentrations of various solutes. Because of a delay associated with the flow of fluid, this regulation tends to become unstable and to produce self-sustained oscillations in nephron pressures and flows. The nephrons interact with one another via two different mechanisms, both mediated by phenomena in the connecting vascular network. Experiments on rats show that neighboring nephrons in the surface of the kidney can synchronize their pressure and flow oscilla-

\footnotetext{
${ }^{a)}$ Corresponding author. Telephone: (401) 863-1619. Fax: (401) 863-1971.

Electronic mail: marsh@ash.biomed.brown.edu
}

tions. It has not yet been possible to perform similar measurements for nephrons deeper in the kidney. Using available anatomical and physiological information we have constructed a model of 22 interacting nephrons, including surface as well as deep nephrons. The model shows that, for physiologically reasonable parameter values, the deep nephrons do not synchronize with the superficial nephrons even though they are coupled via the same blood supply. This lack of synchronization introduces an asymmetry into the system that may be responsible for the development of chaotic dynamics in the tubules of rats with experimental hypertension.

\section{INTRODUCTION}

The interaction between the kidneys and the cardiovascular system is complex. As a part of its role in maintaining body fluid volumes, the kidneys, for instance, produce a set of hormones that affect blood vessels within the kidneys and elsewhere in the body. These hormones may influence the long term structure of the cardiovascular system. Disturbances of kidney function can cause hypertension, a prevalent disease in industrialized societies. There is now a detailed understanding of how the hormones produced in the kidney affect the blood pressure. 
The kidneys are also perfused with blood and are thus exposed to all of the fluctuations present in the cardiovascular system. There are two major periodicites in the blood pressure: one at the frequency of the heart rate $(1 \mathrm{~Hz}$ in humans, $4-6 \mathrm{~Hz}$ in the rodents that are often used for experimental work), and the other with the period of $24 \mathrm{~h}$. Over the intermediate bandwidth between these periodicites the blood pressure displays a $1 / f$ pattern. ${ }^{1,2}$ The $1 / f$ fluctuations are caused by the independent actions of arterioles in all organs of the body, with contractions of large muscles making particularly strong contributions, and they present a specific challenge to the kidneys as they serve to regulate the body fluids.

The process of urine formation begins with the filtration of fluid at the glomerulus, a collection of capillaries that operates at higher vascular pressures than capillaries elsewhere in the body. The formation of glomerular filtrate requires this higher pressure. A small gradient of hydrostatic pressure drives the filtrate along the kidney tubule, also known as the nephron. During this transit, cells of the tubule selectively reabsorb solutes and water. After passage through several successive tubule segments about $80 \%$ of the glomerular filtrate and about $90 \%$ of the filtered $\mathrm{NaCl}$ will have been returned to the blood. At this point the tubule makes contact with the same glomerulus from which it began. The contact point is a structure, the macula densa, specialized for information transfer between the tubule and the arterioles whose vascular resistance determines the blood flow to the nephron.

Epithelial transport processes responsible for body fluid regulation work on the remaining solutes and water, under hormonal control from other organs, including the brain, the adrenal gland, and the kidney itself. The hormonally sensitive sites have limited dynamic range and to be effective in regulating body fluids, the solute load delivered to them in the tubular fluid must remain within relatively narrow bounds. The $1 / f$ fluctuations are large enough that, if unopposed, they could impose large fluctuations on glomerular filtration rate and therefore on the solute load delivered to the hormonally sensitive sites. Such fluctuations could easily become too large to permit the hormones to exert their needed effects.

Kidneys autoregulate their blood flow in response to these fluctuations. The process of autoregulation makes use of the tubular contact with the glomerulus, so that the concentration of electrolytes becomes a controlled variable. Individual nephrons in mammalian kidneys regulate their own blood flow with two mechanisms: tubuloglomerular feedback (TGF), which senses flow rate dependent changes in concentrations of certain electrolytes in the tubular fluid; and myogenic mechanism, which senses vascular hydrostatic pressure in the afferent arterioles. Both adjust the contractile state of vascular smooth muscle in the arteriolar wall to vary vascular resistance to blood flow. Nephrons also communicate with each other by means of vascular signaling initiated by the TGF mechanism and propagated electrotonically and decrementally over the vascular wall. ${ }^{3-6}$ Both TGF regulation and the myogenic mechanism are oscillating nonlinear systems that interact with each other within single nephrons $s^{3,7-13}$ and, by virtue of the vascularly propagated signals, between neighboring nephrons. ${ }^{14,15}$ These interactions produce modulation of the myogenic mechanism by TGF as well as different forms of synchronization. ${ }^{11,12,14}$ So far, these phenomena have been studied experimentally only in cortical nephrons with tubular and vascular components on the surface of the kidney, but because the deeper nephrons have similar structures and regulatory mechanisms, we assume that they, too, generate oscillations and are capable of interactions. If all or most nephrons oscillate, and if they interact, synchronization and other forms of nonlinear interactions are to be expected among nephrons along the same cortical radial artery.

Nephrons that lie deep within the kidney are longer than those on the surface, and because the TGF signal depends on flow rate dependent concentration changes reaching a tubular sensing site, one expects that the longer nephrons will oscillate more slowly than the shorter superficial ones. While interactions among nephrons of different lengths are to be anticipated, the resulting coupling phenomena cannot be predicted in advance.

Using available anatomical and physiological information, we have simulated an ensemble of nephrons of different lengths, supplied from a single artery, and allowed to interact with each other. The questions at issue are whether synchronization occurs, and if it does, whether all nephrons synchronize to a single common frequency. Tubular pressure fluctuations from animals with chronic hypertension are not periodic, and the time series display characteristics of deterministic chaos. ${ }^{16-18}$ In particular, the data sets have positive first Lyapunov exponents and the correlation dimensions increase several fold when the phase values of Fourier transforms of the time series are randomized. Efforts have been made with various models of the system to simulate this transition. However, the values of the TGF feedback gain required to produce chaotic phenomena in existing singlenephron models exceed realistic physiological values by nearly $30 \%$. We wish to use the simulation to test whether the interactions with other nephrons in a vascularly coupled ensemble could promote the transition.

\section{NEPHRON TREE MODEL}

Our model includes both vascular and nephron components. The interactions among the nephrons occur via vascular signal propagation and due to oscillations in vascular pressure within the cortical radial artery. We make use of a relatively simple model of nephron dynamics ${ }^{19,20}$ and consider a tapering cortical radial artery whose dimensions are based on recent measurements made in rat kidneys. ${ }^{24}$

\section{A. Vascular model}

From measurements of signal strength in nephronnephron communication we estimate that the distance over which such signals can be effective is about $1.5 \mathrm{~mm} .^{21,22}$ The renal cortex is about $2 \mathrm{~mm}$ deep in rats, and the cortical radial artery branches from the arcuate artery and penetrates toward the renal surface within the cortex. We assume, therefore, that the field of interacting nephrons consists of all 


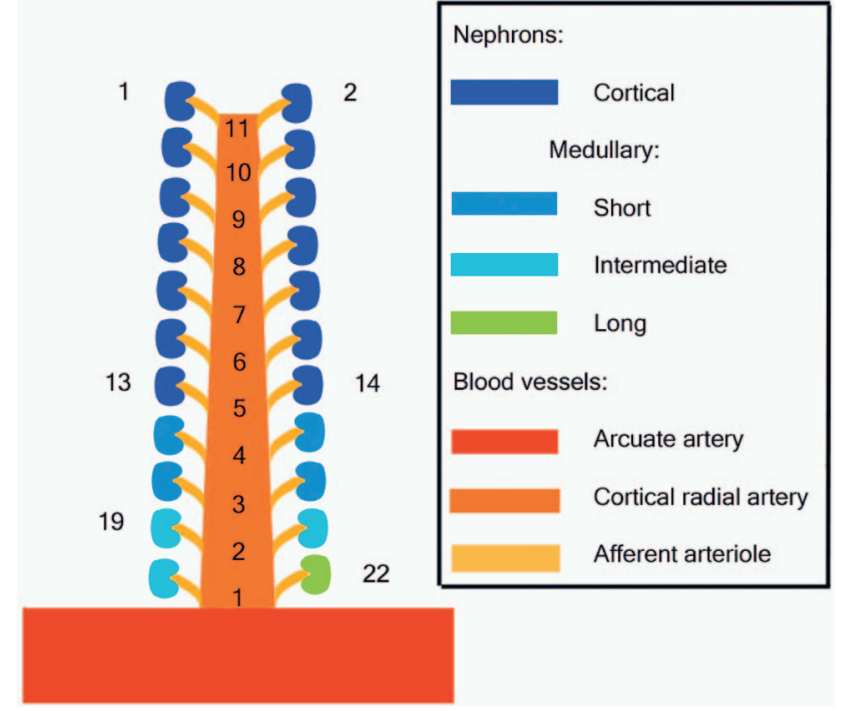

FIG. 1. (Color) Structure of the vascular tree that was modeled; nephrons are depicted by showing their glomeruli. Blood flows from the arcuate artery into the cortical radial artery. Twenty two nephrons receive blood through afferent arterioles from the cortical radial artery. Nephrons are numbered laterally, and the vascular nodes of origin of the afferent arterioles are numbered within the cortical radial artery.

those with afferent arterioles originating from a single cortical radial artery, which includes superficial (cortical) as well as deep (juxtamedullary) nephrons. The number of nephrons originating from a single cortical radial artery varies somewhat; estimates are in the range of $20-40 ;{ }^{23,24}$ we used 22 , of which 14 were defined as cortical and 8 as medullary.

Figure 1 shows the anatomic basis for the vascular model. A tapered cortical radial artery branches from an arcuate artery. One pair of afferent arterioles branches from the arcuate artery and ten pairs from the cortical radial artery. The level of origin of each pair is designated a node. At the ninth node, for example, conservation of blood flow yields

$$
\frac{P_{8}^{b}-P_{9}^{b}}{R_{8}^{b}}-\frac{P_{9}^{b}-P_{10}^{b}}{R_{9}^{b}}-\frac{P_{9}^{b}-P_{5}^{g}}{R_{5}^{g}}-\frac{P_{9}^{b}-P_{6}^{g}}{R_{6}^{g}}=0,
$$

where the superscript $b$ refers to the branching arteriole, and the superscript $g$ to the glomerulus. The values of $R_{k}^{b}$ are calculated as $8 \eta l_{k} / \pi r_{k}^{4}$, using a blood viscosity $\eta$ of 4.6 cPoise ( 1 cPoise $\left.=10^{-3} \mathrm{~kg} / \mathrm{m} \mathrm{s}\right)$, a segment length of $0.02 \mathrm{~cm}$, and radius values $r_{k}$ taken from the literature. ${ }^{24}$ Values of $P^{g}$ and $R^{g}$ are calculated in the nephron model. This formulation (1) is based on the assumption that the vascular tree can be described as purely resistive and that short term, local accumulation of blood can be neglected.

The contribution to the activation of the smooth muscle cells in the arterial wall of the $j$ th nephron produced from vascularly propagated signals from the neighboring nephrons, $\Delta \Psi_{j}$, is modeled by

$$
\Delta \Psi_{j}=\sum_{i=1, i \neq j}^{N} \theta \Psi_{i} \exp \left(-\lambda\left(L_{j i}+2 L^{g}\right)\right),
$$

where $\Psi_{i}$ is the TGF activation potential of TGF for the $i$ th nephron as calculated from the nephron model. $L_{j i}$ is the distance from the origin of the $j$ th to the origin of the $i$ th afferent arteriole along the cortical radial artery; $L^{g}$ is the length of the afferent arteriole, assumed identical for all nephrons; and $\lambda$ is a length constant characterizing the exponential decrease of the vascular propagated signal along the blood vessels. $\theta$ is a control parameter that we use to examine the effects of different coupling strengths. $\theta=0.25-0.50$ is considered the range of physiologically realistic values for animals with normal blood pressure. Twice the value of $L^{g}$ is used because the TGF signal must be propagated down the afferent arteriole of the TGF mechanism that initiates the signal, and up the afferent arteriole of the TGF mechanism receiving it. Equation (2) expresses the current state of knowledge of the interaction; a change in the TGF signal in one nephron can be detected in the TGF mechanism of a neighboring nephron, and the effect of the interaction decreases exponentially with the vascular distance the signal must traverse. ${ }^{6,21,22}$ The mechanisms underlying these interactions are presently not known in detail and we offer no hypothesis regarding them.

\section{B. Nephron model}

We used a model developed by the Copenhagen groups ${ }^{19,20}$ to represent each of the 22 nephrons. The model has been described and evaluated in detail in other studies, ${ }^{5,14,19,20,25}$ and is presented unmodified in the Appendix. The model consists of a set of five coupled ordinary differential equations. One equation expresses the rate of change of the proximal tubule pressure as a function of glomerular filtration rate, rate of proximal tubule reabsorption, and outflow from the proximal tubule into the loop of Henle; another is a second order equation expressing the rate of change of the afferent arteriolar radius as a function of vascular pressure and TGF input; and the remaining three equations constitute a third order delay designed to simulate the transit of tubular fluid through the tubule to the macula densa; epithelial transport of $\mathrm{NaCl}$ in a tubular segment, the thick ascending limb of Henle's loop, that is responsible for the flow dependent variation of $\mathrm{NaCl}$ concentration at the tubular sensing site; and the time for signal propagation from the macula densa to the afferent arteriole.

A number of studies report measurements of oscillations of tubular pressure from cortical nephrons on the surface of the rat kidney. The period length of the TGF oscillation is $30-50 \mathrm{~s}$. The nephron model used in this study requires a value for the time delay of $12 \mathrm{~s}$ to reproduce a $30 \mathrm{~s}$ period, and we have used this value as the starting point for all calculations with cortical nephrons. There are no measurements of proximal tubule pressure in juxtamedullary nephrons. We have used a more detailed model ${ }^{11}$ to estimate the effect of increasing tubular length on the oscillation period. The inner medulla in the rat is $0.5 \mathrm{~cm}$ long. Adding descending and thin ascending limbs to this length increases the period about $50 \%$. Different nephrons penetrate to intermediate depths of the inner medulla. The increase in period is likely to vary in proportion to the length of the segment. The inner medulla is conical in the rat, and the density of medullary nephrons is approximately constant over the volume of the inner medulla. This suggests that the number of nephrons decrease with increasing depth. In addition to the 14 
cortical nephrons, we have therefore used four medullary nephrons that turn at a depth of $0.1 \mathrm{~cm}$ in the inner medulla, three nephrons that reach $0.3 \mathrm{~cm}$, and one nephron that reaches a depth of $0.5 \mathrm{~cm}$. The assumed time delays were $14.0 \mathrm{~s}, 16.3 \mathrm{~s}$, and $17.6 \mathrm{~s}$, respectively, and $12.0 \mathrm{~s}$ for the cortical nephrons.

An important issue we wish to address in this paper is to what extent synchronization occurs among nephrons in the ensemble. If all the cortical nephrons have a single value of the time delay, and all the short medullary nephrons another single value, and so on, the question may be relatively simple. We have therefore introduced variation in the time delays by adding random numbers to the time delay in each of the 22 nephrons. The distribution of random numbers was Gaussian with a zero mean and a standard deviation of $0.4 \mathrm{~s}$. A fixed value of the delay was used in each nephron for each run.

The nephron models were solved with a fourth order Adams Moulton predictor-corrector method using a fixed time step of $1 \times 10^{-4} \mathrm{~s}$. All nephron models were solved at each time step, and the pressure at each of the nodes in the cortical radial artery was calculated. The set of calculations was then iterated until the Euclidian norm of the vector of vascular pressures varied less than $1.0 \times 10^{-6}$ on successive iterations. Two or three iterations were sufficient to achieve this level of convergence.

\section{SIMULATION RESULTS}

The model produced a mixture of steady state, quasiperiodic, and chaotic solutions for nephrons in the ensemble. The value of the tubule pressure in the steady state solutions varied with arterial pressure and the strength of nephronnephron coupling. Figure 2 shows examples of phase plane projections of the quasiperiodic and chaotic solutions, together with the results of a simulation of a single nephron not a part of an ensemble. The results are shown as trajectories on the plane defined by the tubular hydrostatic pressure and the afferent arteriolar radius.

Our results for a single nephron, shown in Fig. 2(a), are the same as have been reported previously. ${ }^{5,14,19,25}$ The tubule pressure and the arteriolar diameter oscillate, and the motion on the phase plane is that of a limit cycle oscillator. Parameters have been chosen that are typical of measurements made in normal animals. With these values, $\alpha=12$ and arterial pressure $100 \mathrm{~mm} \mathrm{Hg}$, the myogenic oscillations remain inactive in the dynamics of the uncoupled nephron. The value of $\alpha=12$ was used for all simulations reported in this study. For higher values of the gain parameter $\alpha$ of the TGF mechanism, the individual nephron displays a combination of fast myogenic and slower tubuloglomerular oscillations and, as previously reported, one can observe the phenomena of intranephron synchronization, period-doubling and transitions to chaos. ${ }^{14,19}$

The simulation of the nephrons that form the ensemble behaved differently in several respects. First, the amplitude of the tubular pressure fluctuation was greater in nephrons acting as part of the ensemble than in the single nephron, even though the parameter values were the same in the respective simulations. This result is analogous to the report by
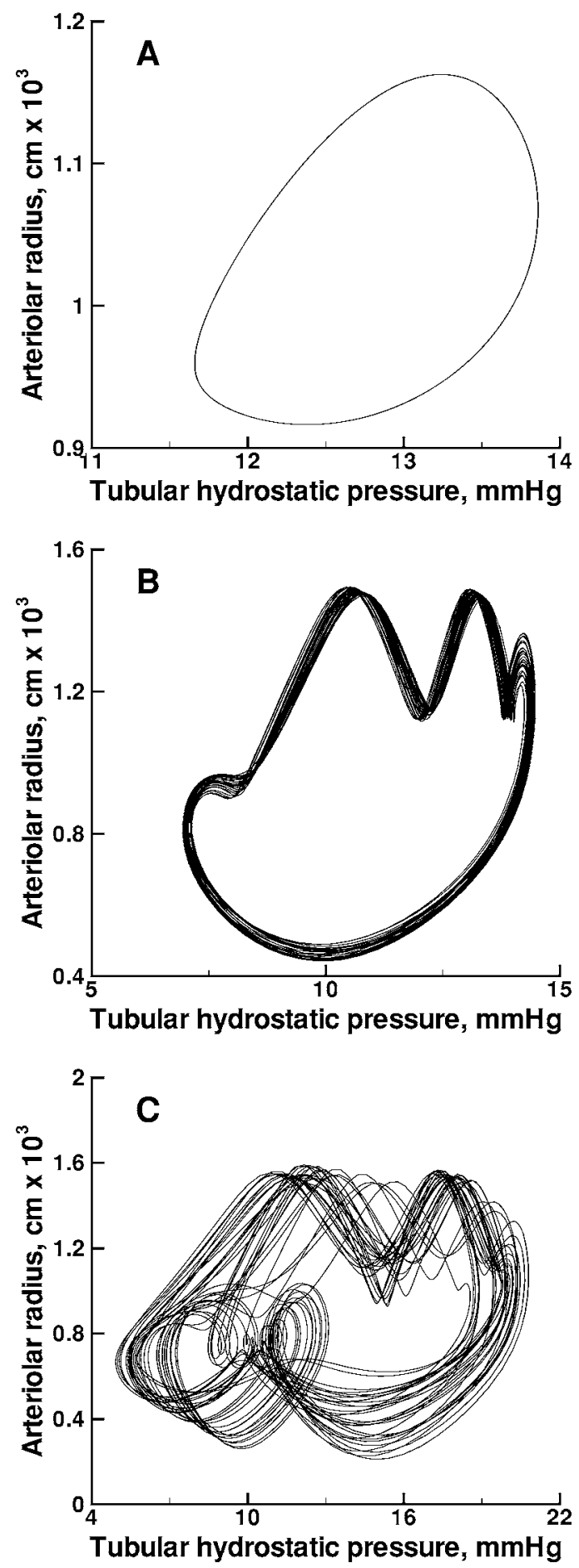

FIG. 2. Phase plane plots of nephron model results. Panel A: a single nephron model, showing a limit cycle oscillation. Panel B: nephron 1 of the ensemble of 22, with arterial pressure at $100 \mathrm{~mm} \mathrm{Hg}$ and $\theta$, the strength parameter of nephron-nephron coupling, at 0.5, showing quasiperiodic motion. Panel C: nephron 1, with arterial pressure at $125 \mathrm{~mm} \mathrm{Hg}$ and $\theta$ at 1.0 , showing a chaotic attractor. Each figure was obtained by simulating $5000 \mathrm{~s}$.

Pitman et $a l^{26}$ that the value of the bifurcation parameter needed to achieve the Hopf bifurcation in a single nephron model was reduced when two nephron models were coupled. Second, the simulations produced almost periodic behavior in many nephrons, but in no case was the motion strictly that of a limit cycle oscillator. As shown in Fig. 2(b), one form of 


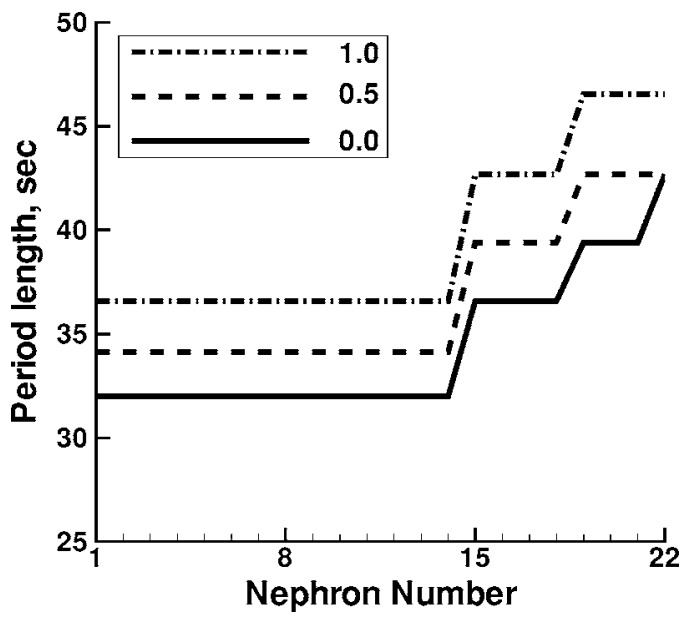

FIG. 3. Period length vs nephron number, for three values of $\theta$, the strength of coupling between nephrons. Arterial pressure: $100 \mathrm{~mm} \mathrm{Hg}$.

behavior showed quasiperiodicity, with two or more incommensurate frequencies.

A typical power spectrum from a nephron with quasiperiodic oscillations shows several peaks: three strong peaks associated with the TGF signal and its harmonics, peaks at higher frequencies arising from the myogenic oscillations and their harmonics, and a broader less pronounced peak at very low frequencies arising from the interactions in the nephron tree. Similar low frequency components have been observed in experimental spectra and ascribed to internephron coupling phenomena, presumably in the form of beating between the TGF oscillations in different nephrons or because of a new type of collective modes in the tree structure. $^{27}$

For purposes of assessing synchronization we have compared only the frequencies of the TGF oscillation; we considered neither the fast myogenic oscillations nor the slower frequencies imposed by the ensemble. We implemented the model under two sets of conditions designed to permit study of different aspects of synchronization. To test the interaction among nephrons of different lengths we applied identical time delays to all cortical nephrons, and longer delays to the three groups of medullary nephrons, as described above. In the other set we added small random contributions to the time delay of each of the nephrons.

We first examined nephrons operating only in a quasiperiodic mode, which occurs at an arterial pressure of $100 \mathrm{~mm} \mathrm{Hg}$ and a coupling parameter of $\theta=0.5$. These values are the normal arterial pressure and the value of $\theta$ measured experimentally in normotensive rats. Figure 3 shows the dominant period lengths for various values of the coupling strength. In the data set with no nephron-to-nephron coupling, the period lengths for cortical nephrons did not vary from nephron to nephron because the time delays were identical.

Increasing nephron-to-nephron coupling to encompass the range of physiologically measured values had several effects. Period lengths for all nephrons increased with coupling strength, and, as will be seen below, the amplitude of tubular pressure oscillations increased. Increased coupling also per- mitted synchronization among juxtamedullary nephrons with different time delays. Nephron 22 has a longer delay than any of the others, and has the longest period length when internephron coupling is set to 0 . At higher values of the coupling strength, nephron 22 becomes entrained with nephrons 19-21 to form a cluster with a single frequency. The shorter juxtamedullary nephrons, numbers $15-18$, all oscillate at a single frequency, which did not become entrained with either the cortical nephrons or the longer juxtamedullary ones. The model therefore predicts at least three distinct frequencies operating along a cortical radial artery.

Next we altered the time delay in each of the 22 nephrons by adding a random number as described above. As shown in Fig. 4(a), randomization of the time delays produces a set of time series without synchronization, so long as the coupling strength $\theta$ was 0 . The juxtamedullary nephrons, numbers $15-22$, had longer periods and larger oscillations than the cortical nephrons.

Figure 4(b) shows the same array of nephrons as in Fig. 4 , upper panel, but with the value of $\theta$ set to 0.5 , which falls within the range of experimentally measured values, and that permits synchronization. The magnitude of the oscillations was greater with synchronization than without. There were phase differences among the shorter cortical nephrons, but all oscillated at the same frequency. The juxtamedullary nephrons did not synchronize with cortical nephrons. The introduction of variation in the time delays produced a more complex pattern of synchronization among the juxtamedullary nephrons than is shown in Fig. 2, and this pattern differed among the sets produced by different random number series. In all cases the cortical nephrons synchronized among themselves at a single frequency, but in no case did the juxtamedullary nephrons synchronize with the cortical nephrons.

Results with a parameter set selected to generate a chaotic time series in all nephrons, arterial pressure at $125 \mathrm{~mm} \mathrm{Hg}$ and $\theta=1.0$, are shown in Fig. 4(c). Although all 22 time series in this simulation showed attractors similar to those of Fig. 1(c), the dominant frequency was that of the TGF oscillation, and each of the oscillations had the same frequency, demonstrating strong synchronization.

Figure 5, first panel, shows the pressures in the cortical radial artery from the same simulation shown in Fig. 4, first panel, with no nephron-nephron coupling. The pressure drop was calculated using vessel length and radius data from Ref. 24. There is a pressure drop of a few percent along the cortical radial artery as it distributes blood to the afferent arterioles, and there are minor oscillations in pressure. Figure 4, first panel shows the tubular pressure oscillations present in the nephrons associated with the vascular pressures in Fig. 5, first panel. The tubular pressures remain unsynchronized. This result suggests that hemodynamic coupling is not functionally important under the present conditions.

Figure 5, second panel, shows vascular pressure results from the same simulation shown in Fig. 4, second panel, with nephron-nephron coupling active. When there is synchronization of nephron oscillations an oscillation in vascular pressure emerges at the same frequency as the synchronized oscillation of the cortical nephrons. There is an 


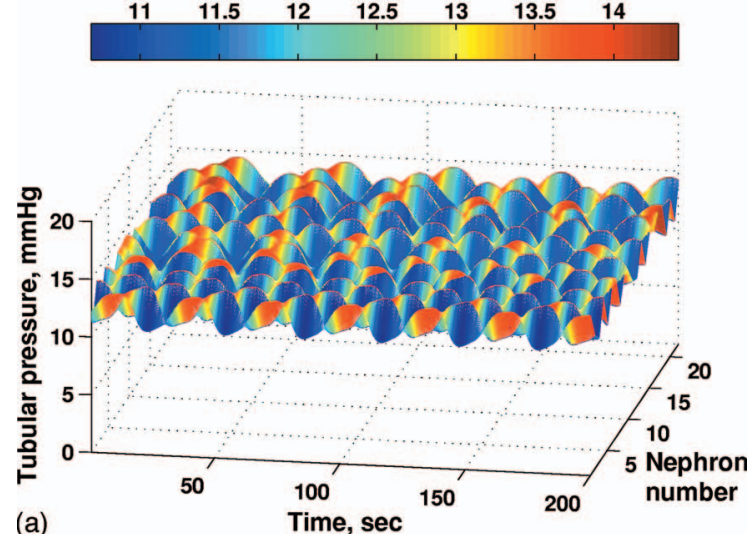

(a)
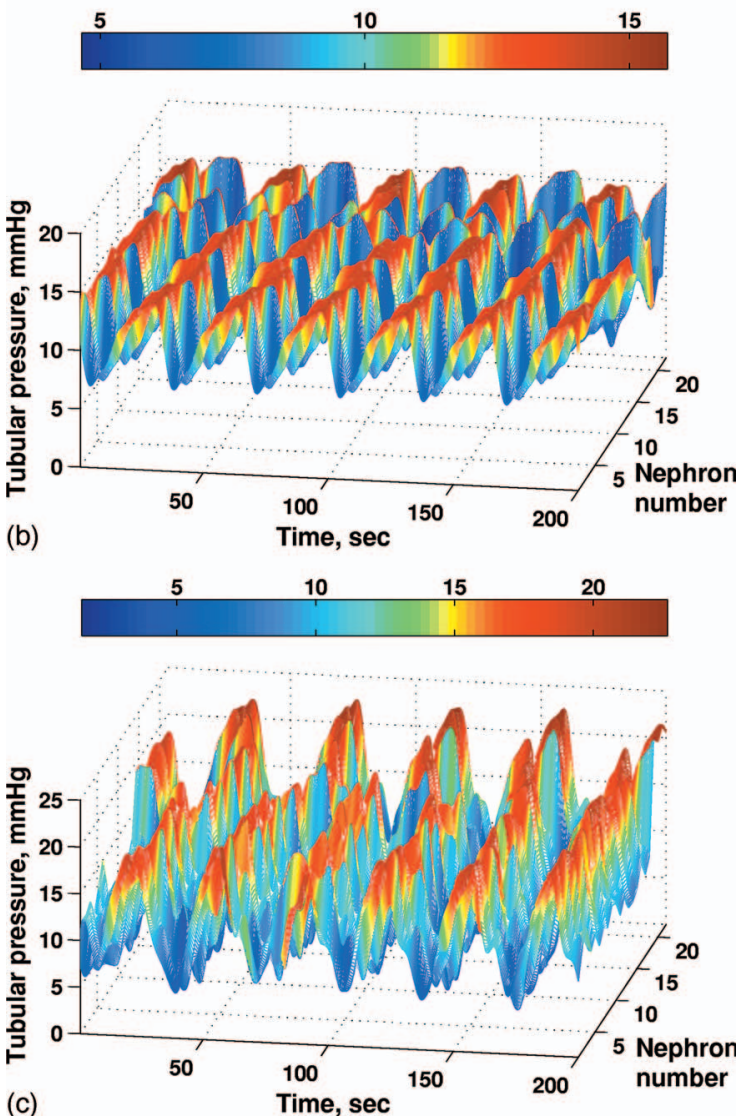

FIG. 4. (Color) Tubular hydrostatic pressure in all nephrons of the ensemble. First panel: $\theta=0$; arterial pressure: $100 \mathrm{~mm} \mathrm{Hg}$. Second panel: $\theta$ $=0.5$; arterial pressure: $100 \mathrm{~mm} \mathrm{Hg}$. Third panel: $\theta=1.0$; arterial pressure: $125 \mathrm{~mm} \mathrm{Hg}$. The bars show the color scales used to present the tubular pressures in the three panels.

imperceptible oscillation of the vascular pressure supplying the longer juxtamedullary nephrons. These longer nephrons are supplied from the arcuate artery and the beginning of the cortical radial artery where the blood flow is higher than at the end of the cortical radial artery. Because the blood flow in the cortical radial artery near its beginning is large compared to the blood flow to the medullary nephrons, the oscillations in blood flow to these nephrons have only minor effects on vascular pressure. As blood flows toward the end of the cortical radial artery the blood flow rate drops and the effect of oscillations in afferent arteriolar resistance increase, leading to larger oscillations in vascular pressure.
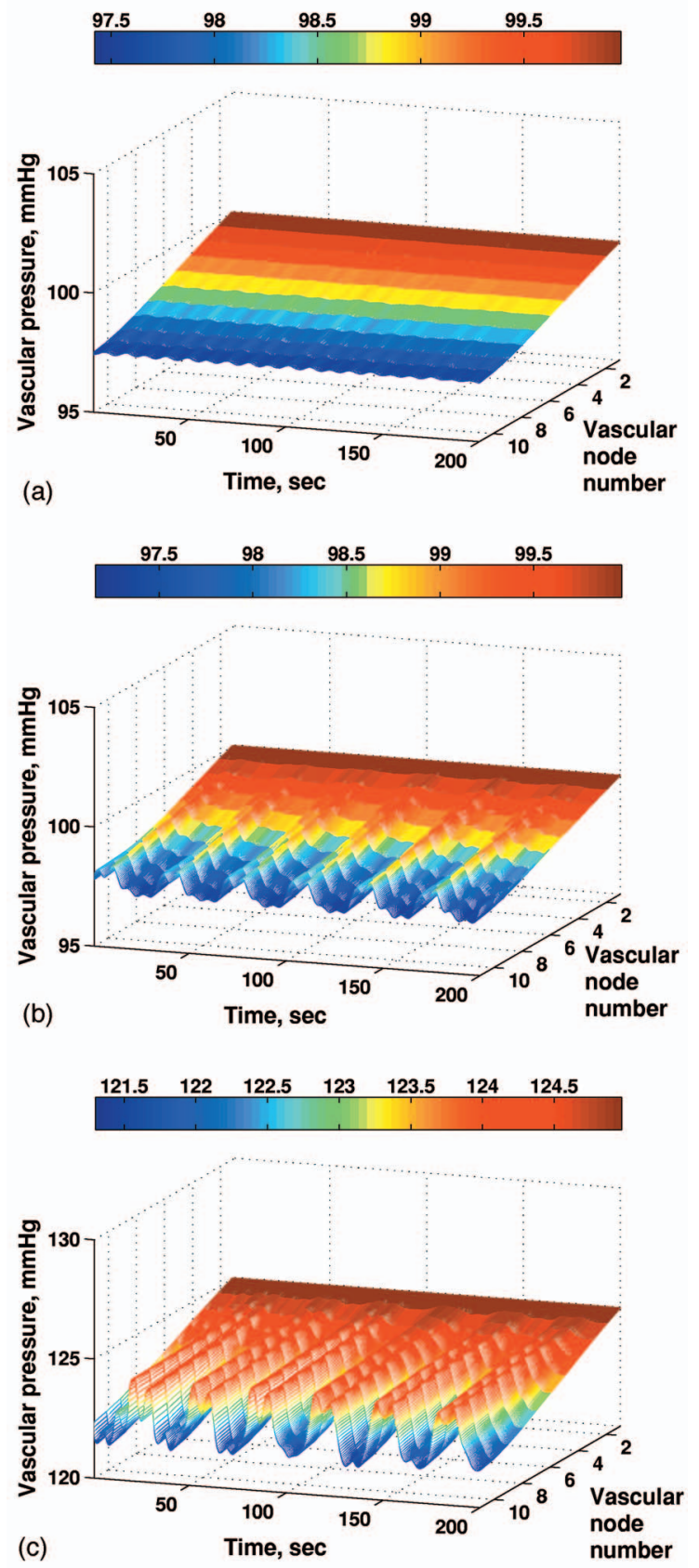

FIG. 5. (Color) Vascular hydrostatic pressure in the model. Top panel: $\theta$ $=0$, arterial pressure: $100 \mathrm{~mm} \mathrm{Hg}$. Middle panel: $\theta=0.5$, arterial pressure: $100 \mathrm{~mm} \mathrm{Hg}$. Lower panel: $\theta=1.0$, arterial pressure: $100 \mathrm{~mm} \mathrm{Hg}$.

Figure 5, third panel, contains the results of vascular pressure calculations in the data set of Fig. 4, third panel, that predicts chaotic tubular pressure trajectories. An oscillation in vascular pressure accompanies the oscillation in tubular pressures and flows, and in addition, the irregular fluctuations that give the tubular pressure time series their chaotic property produce similar irregularities in the vascular pressure record. The oscillation in vascular pressure shown in Figs. 5(b) and 5(c) arise because of resource distribution by the network.

To judge the effectiveness of the internephron signaling on synchronization, we ran 12 simulations using unique seed values for the random number generator, once with coupling 

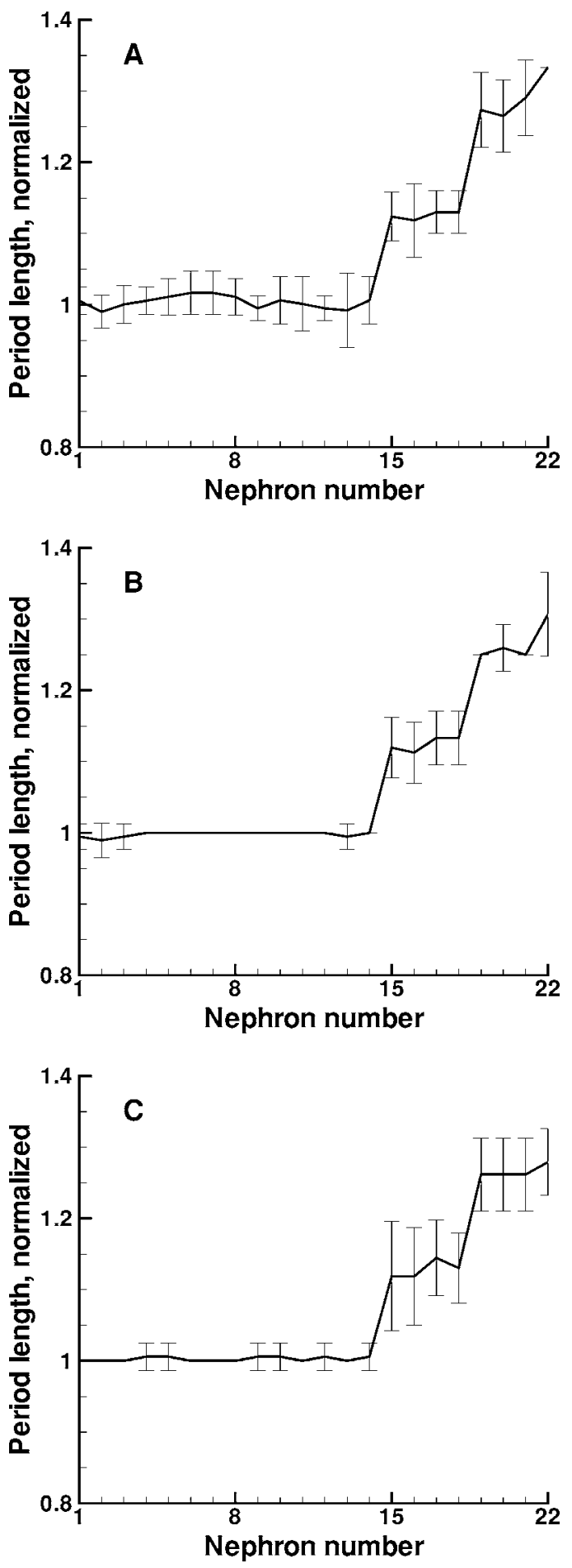

FIG. 6. Period length in the 22 nephrons with varying time delays and arterial pressures. Panel A: $\theta=0.0$. Panel B: $\theta=0.5$, both at arterial pressure $100 \mathrm{~mm} \mathrm{Hg}$. Panel C: $\theta=1.0$ and arterial pressure $125 \mathrm{~mm} \mathrm{Hg}$. Data presented as the mean of 12 simulations $\pm \mathrm{SD}$. In panel $\mathrm{B}$, the standard deviation values were 0 at positions $4-12,14,19$, and 21 . In panel $\mathrm{C}$, standard deviation values were 0 at positions $1-3,6-8,11$, and 13 .

set to zero, once with coupling at $\theta=0.5$, and once with coupling at 1.0 and blood pressure elevated. Figure 6 shows the results. In each of the 12 simulations, the period length in each nephron was normalized by the modal period length of cortical nephrons in that simulation. Without coupling there is variation in the frequencies of the nephrons at each posi- tion. Activation of coupling eliminates the variation at 11 of the 14 cortical nephrons, as shown in Fig. 6(b). The reduction in the variance with coupling is evidence of strong synchronization, because the interaction leads to changes in frequency, and convergence on a common frequency. When the parameter set forces the system to adopt a set of chaotic trajectories, there is also a reduction in the variance of the cortical nephrons, as shown in Fig. 6(c). In 11 of the 12 simulations there was complete synchrony at a single frequency among all the cortical nephrons. That one exception is responsible for all of the variance in cortical nephrons in Fig. 6(c).

The juxtamedullary nephrons showed comparable variation in all three sets of simulations. The formation of synchronized clusters among juxtamedullary nephrons is sensitive to their time delays, and varied from one simulation to the next. This variation is reflected in persistence of high values of the standard deviation despite the use of a normal value for the coupling strength. Nevertheless, the results shown in Fig. 6 are similar to those shown in Fig. 3, which leads us to conclude that randomization of nephron lengths does not destroy the clustering properties of the network.

TGF and the myogenic mechanism work in the kidney to regulate blood flow to individual nephrons; the main perturbations come from the arterial pressure, which fluctuates with a $1 / f$ pattern. ${ }^{1,2}$ We therefore exercised the model varying blood pressure over the range 90 to $130 \mathrm{~mm} \mathrm{Hg}$, and the coupling parameter $\theta$ from 0.0 to 1.5 . We sought only to determine the main classification of dynamical activity over these ranges. Figures 7(a)-7(c) present the results for nephrons 1,15 , and 22 , respectively. Nephron 1 is the most superficial of the cortical nephrons and represents those on the surface of the kidney that have been submitted to experimental investigation. Nephron 15 is the shortest of the juxtamedullary nephrons; its dynamics have not been studied experimentally. Nephron 22 is the longest juxtamedullary nephron, and its dynamics have also not been studied experimentally. A single value of the seed for the random generator was used for all of the 110 simulations represented in Fig. 7.

In all cases these nephron simulations retain a strong component at the TGF frequency. As can be seen in all panels the complete absence of nephron-nephron communication generates oscillations over a range of arterial pressures from 93 to $103 \mathrm{~mm} \mathrm{Hg}$. On both sides of this interval the model generates steady state behavior for all nephrons. The arterial pressure here is either too low or too high for the TGF regulation to be active, and the nephrons operate in one of the saturation regimes for the S-shaped curve (A2) determining the activation of the arteriole smooth muscles. Quasiperiodicity appears over intermediate ranges of $\theta$ and arterial pressure for all nephrons. The fraction of the plane covered by quasiperiodic behavior is maximal in nephron 1 and diminishes in deeper and longer nephrons. For the rest there are trajectories that form strange attractors on the phase plane.

The simulations in Fig. 7(a) represent the behavior of the most superficial cortical nephrons from which the available experimental results have been collected. In animal experiments the arterial pressure is easily changed, but the value of 
A

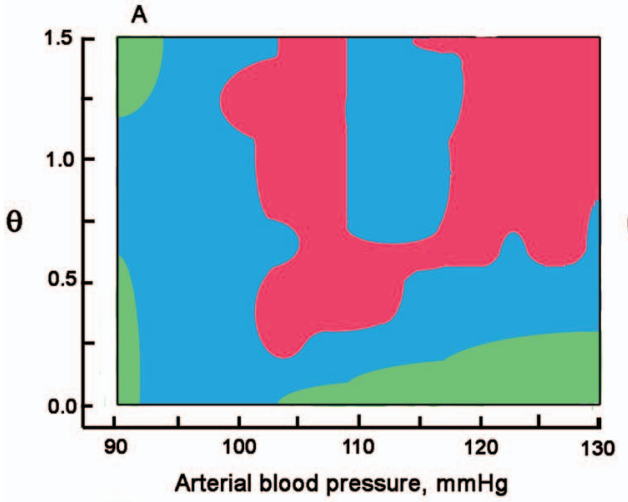

B

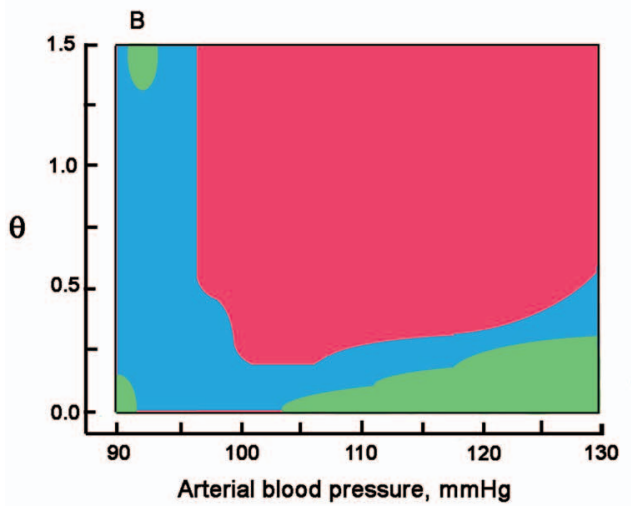

C

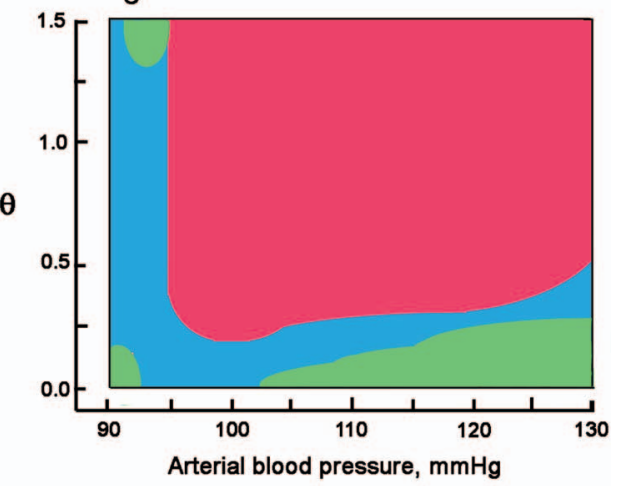

FIG. 7. (Color) Dynamical behavior regimes as a function of arterial pressure and $\theta$, the strength of nephronnephron coupling. Panel A: nephron 1, the most superficial cortical nephron; panel B: nephron 15, the shortest juxtamedullary nephron; and panel $\mathrm{C}$ : nephron 22, the longest juxtamedullary nephron. $\theta$ is not. The value of this parameter is in the range $0.25-0.5$ in normal animals. ${ }^{21,22}$ As mentioned above, oscillations in tubular pressure are typically measured in normal rats over the arterial pressure range $95-130 \mathrm{~mm} \mathrm{Hg}$. Figure 7(a) shows a region of chaotic behavior in the arterial pressure range $100-115 \mathrm{~mm} \mathrm{Hg}$ when $\theta$ lies between 0.25 and 0.5 . Results such as these have not been reported in animal experiments that have concentrated precisely in this area of the parameter plane. We address possible sources of this discrepancy in the Discussion.

\section{DISCUSSION}

We simulated the dynamical behavior of a group of nephrons that derive their blood supply from a single cortical radial artery in a rat kidney. The simulations include simplified representations of the adaptive mechanisms present in every nephron that regulate blood flow. The cortical radial artery is an example of a resource distribution network. ${ }^{25}$ All the blood flowing into it is distributed to the nephrons whose arterioles branch from it, with none remaining at the end. The cortical radial artery is relatively large compared to the arterioles, and we assumed that active control of blood flow is exercised by the nephrons, and none by the artery. In addition to local regulation of blood flow, nephrons communicate with each other by sending electrical signals over the vascular wall. The signals are initiated by TGF, the mechanism that provides local regulation of blood flow, and influences the vascular response of adjacent nephrons. ${ }^{4,6,21,22}$

We used the simulation to address several questions. First, is nephron-to-nephron communication sufficient to explain the strong synchronization of tubular pressure oscillations found in normal rats and those with hypertension. ${ }^{4,17,18}$
Tubular pressure in normal rats oscillates; in hypertensive rats the fluctuations are irregular and have characteristics consistent with deterministic chaos. ${ }^{16-18}$ In the simulation all of the short cortical nephrons synchronize at a single frequency, even when the time delays in the TGF loop are made to vary randomly, and also when the cortical nephrons operate in a chaotic domain. The longer medullary nephrons consistently form two different clusters each operating at a unique frequency, and neither synchronizing with the cortical nephrons.

Next, what is the relative significance of different coupling mechanisms? Two types of nephron-to-nephron communication have been discussed: by electrical signalling over the vascular wall, and by modifying the local hydrostatic pressure in the cortical radial artery, called hemodynamic coupling. ${ }^{25,28}$ Hemodynamic coupling predicts out of phase oscillations in adjacent nephrons, but it can only be effective if the contraction of a single afferent arteriole can make an appreciable difference in the vascular pressure. Our simulation results show that adjacent cortical nephrons oscillate at a single frequency and with small phase angle differences. The effect of electrical coupling over the vascular wall overwhelms the possible effect of hemodynamic coupling.

The third question relates to the limitations of the present model. TGF provides an S-shaped response to flow in the loop of Henle [Eq. (A2)], and direct measurements show that the mechanism saturates at either high or low flow rates with a narrow range of pressures over which effective regulation is possible. This result is not consistent with other experimental results; higher arterial pressures generate larger oscillations. ${ }^{13}$ Two explanations for this discrepancy come to mind: coupling within the nephron ensemble is responsible, 
or the model is too simple. A more detailed nephron model, which has a spatially distributed renal tubule, and a contractile mechanism in afferent arteriolar cells shows oscillations in both TGF and the myogenic mechanism, and the amplitudes of the oscillations increase monotonically with arterial pressure over the range $95-130 \mathrm{~mm} \mathrm{Hg} .{ }^{11}$

At higher arterial pressures and higher values of coupling strength each of the nephrons in the ensemble is capable of a bifurcation to chaos. The longer medullary nephrons appear from the simulation results to operate with chaotic dynamics under most combinations of parameters and arterial pressure that are found in normal animals. The time series generated by these simulations retain the TGF periodicity and strong synchronization that occurs under these circumstances. The simulation suggests that the nephron ensemble has considerable instability under normal circumstances, and that the instability increases at higher arterial pressures and values of the coupling strength, which occurs when blood pressure elevations become chronic.

The simulation predicts some results that are not present in experimental measurements. Figure 7(a) shows a range of chaotic solutions for the most superficial cortical nephrons over an arterial pressure range from 103 to $113 \mathrm{~mm} \mathrm{Hg}$, and a range of values of $\theta$ from 0.25 to 0.50 . These are well within the range of experimental results obtained from normal animals, where only oscillations have been found. Chaos evolves through multiple scenarios in high dimensional dynamical systems. One of the typical routes to chaos, well known through results as well as from experiments, is the transition from quasiperiodic oscillations. ${ }^{29,30}$ The transition from an ergodic torus to chaos can be smooth and related to the loss of smoothness of the torus and even to the appearance of fractal structure without mixing of trajectories. ${ }^{31}$ Whether such transitions occur in the kidney has not been examined, and the inevitable presence of noise may make it impossible to determine with confidence whether there is a quasiperiodic transition to chaos. Alternatively, the model of nephron dynamics we used is quite simple, and the distribution of regimes may change when more realistic models are used.

\section{ACKNOWLEDGMENTS}

This work was partly supported by the European Union through the Network of Excellence BioSim, Contract No. LSHB-CT-2004-005137. D.J.M. acknowledges support from NIH Grant No. EB003508.

\section{APPENDIX: NEPHRON MODEL}

The single nephron model ${ }^{19}$ consists of a set of five coupled differential equations expressing the rate of change of the proximal tubular pressure, the oscillatory dynamics of the afferent arteriolar radius, and the delay associated with the flow of fluid through the loop of Henle. The main nonlinearities of the model are related to the feedback regulation of the arteriolar radius and to the relation between this radius, the degree of activation of the arteriolar smooth muscles, and the equilibrium pressure in the arteriole. Other nonlinearities are associated with the relation between the radius and the flow resistance of the arteriole and between the tubular pressure and the glomerular filtration rate.

The rate of change of tubular pressure $P_{t}$ is given by the rate of change of the fluid volume divided by the tubular compliance $C_{\text {tub }}$,

$$
\dot{P}_{t}=\left(F_{\text {filt }}\left(P_{t}, P_{k}^{b}, r^{b}\right)-F_{\text {reab }}-F_{\text {Hen }}\right) / C_{\text {tub }},
$$

where the incoming flow, the glomerular filtration rate $\mathrm{GFR} \equiv F_{\text {filt }}$ is expressed as a function of the tubular pressure, the pressure of the $k$ th node of the cortical radial artery $P_{k}^{b}$, and the radius of the afferent arteriole $r^{b} . F_{\text {reab }}$ is the rate of tubular fluid reabsorption in the proximal tubule, and $F_{\text {Hen }}$ is the outflow of the tubule into the loop of Henle.

Tubuloglomerular feedback (TGF) exerts its effects on the afferent arteriole, reducing its radius in response to increasing $\mathrm{NaCl}$ concentrations at the macula densa. In the usual experimental evaluation of the TGF mechanism, the flow rate into the loop of Henle is the independent variable, glomerular filtration rate is clamped at 0 , and one measures a change in proximal tubule pressure as a reflection of the resistance of the afferent arteriole. The effect of changes in the loop of Henle flow can be expressed as

$$
\Psi=\Psi_{\max }-\frac{\Psi_{\max }-\Psi_{\min }}{1+\exp \left(\alpha\left(3 X_{3} / T F_{\mathrm{Hen} 0}-S\right)\right)},
$$

where $\Psi_{\max }$ and $\Psi_{\text {min }}$ are the upper and lower activation limits of the TGF mechanism, $\alpha$ is the maximum slope and $S$ is the inflection point of the S-shaped curve that characterizes the TGF relation. $\alpha$ is usually referred to as the TGF feedback gain. $3 X_{3} / T$ is a delayed version of the flow into the loop of Henle and $F_{\text {Hen } 0}$ is a normalized value of the loop of Henle flow.

The afferent arteriole is modeled as a damped second order system

$$
\ddot{r}^{b}+d \dot{r}^{b}=\frac{P_{\mathrm{av}}-P_{\mathrm{eq}}}{\omega},
$$

where, as before, $r^{b}$ is the afferent arteriolar radius, normalized to its resting value, and $P_{\text {av }}$ and $P_{\text {eq }}$ are, respectively, average and equilibrium values of the vascular pressure in the arteriole. $d$ is a damping coefficient, and $\omega$ is a parameter that controls the dynamic characteristics of the myogenic oscillations. The subscript av on the pressure $P_{\text {av }}$ refers to the fact that this pressure is evaluated as the average hydrostatic pressure along the length of the afferent arteriole,

$$
P_{\mathrm{av}}=\frac{1}{2}\left(P_{k}^{b}-\left(P_{k}^{b}-P_{g}\right) \beta \frac{R_{a 0}}{R_{a}}+P_{g}\right)
$$

with the glomerular pressure given by

$$
P_{g}=P_{v}+R_{e}\left(\frac{P_{k}^{b}-P_{g}}{R_{a}}-F_{\text {reab }}\right) .
$$

Equations (A4) and (A5) express simple linear relations between flow rates and pressure drops in the arteriolar system. $P_{v}$ is the venous pressure, and $R_{a}$ and $R_{e}$ are the flow resistances of the afferent and the efferent arterioles. $\beta$ is the fraction of the total afferent arteriolar length that responds to the TGF signal, and $R_{a 0}$ is a resting value of $R_{a}$, 


$$
\begin{aligned}
P_{\text {eq }}= & 0.006 \exp \left(10\left(r^{b}-0.8\right)\right)+1.6\left(r^{b}-1\right) \\
& +\Psi\left(\frac{4.7}{1+\exp \left(13\left(0.4-r^{b}\right)\right)}+7.2\left(r^{b}+0.9\right)\right)
\end{aligned}
$$

is the hydrostatic pressure at which the afferent arteriole is at equilibrium for a given muscular activation $\Psi$ and a given radius $r^{b}$. Terms that are independent of $\Psi$ represent the nonlinear elastic deformation of the vascular wall, and terms proportional to $\Psi$ represents the active (myogenic) response. Equation (A6) was obtained through a combination of theoretical considerations and curve fitting to experimental results.

Regulation of pressures and flows in the nephron is dominated by the negative feedback associated with the TGF mechanism. However, there is a delay in this feedback associated with the time it takes for a flow change to propagate from the glomerulus through the tubule to the macular densa, and with signal transmission from the macula densa cells to the arteriolar smooth muscle cells. The model represents this delay as a third order delay structure,

$$
\begin{aligned}
& \dot{X}_{1}=F_{\text {Hen }}-\frac{3}{T} X_{1}, \\
& \dot{X}_{2}=\frac{3}{T}\left(X_{1}-X_{2}\right), \\
& \dot{X}_{3}=\frac{3}{T}\left(X_{2}-X_{3}\right),
\end{aligned}
$$

where $T$ is the total delay time, and $3 X_{3} / T$ is the delayed flow that serves as an input to the TGF mechanism (A2).

${ }^{1}$ N. H. Holstein-Rathlou, J. He, A. J. Wagner, and D. J. Marsh, "Patterns of blood pressure variability in normotensive and hypertensive rats," Am. J. Physiol. 269, R1230-R1239 (1995).

${ }^{2}$ D. J. Marsh, J. L. Osborn, and A. W. Cowley, Jr., "1/f fluctuations in arterial pressure and the regulation of renal blood flow in dogs," Am. J. Physiol. 258, F1394-F1400 (1990).

${ }^{3}$ K. H. Chon, R. Raghavan, Y. M. Chen, D. J. Marsh, and K. P. Yip, "Interactions of TGF-dependent and myogenic oscillations in tubular pressure," Am. J. Physiol. Renal 288, F298-F307 (2005).

${ }^{4}$ N.-H. Holstein-Rathlou, "Synchronization of proximal intratubular pressure oscillations: Evidence for interaction between nephrons," Pflügers Arch. 408, 438-443 (1987).

${ }^{5}$ N. H. Holstein-Rathlou, K. P. Yip, O. V. Sosnovtseva, and E. Mosekilde, "Synchonization phenomena in nephron-nephron interaction," Chaos 11, 417-426 (2001).

${ }^{6}$ Ö. Källskog and D. J. Marsh, "TGF-initiated vascular interactions between adjacent nephrons in the rat kidney," Am. J. Physiol. 259, F60-F64 (1990).

${ }^{7}$ N.-H. Holstein-Rathlou, A. J. Wagner, and D. J. Marsh, "TGF dynamics and renal blood flow autoregulation in rats," Am. J. Physiol. 260, F53F68 (1991)

${ }^{8}$ N.-H. Holstein-Rathlou and D. J. Marsh, "Oscillations of tubular pressure, flow, and distal chloride concentration in rats," Am. J. Physiol. 256, F1007-F1014 (1989).

${ }^{9}$ N.-H. Holstein-Rathlou, P. Christensen, and P. P. Leyssac, "Effects of halothane-nitrous oxide inhalation anesthesia and Inactin on overall renal and tubular function in Sprague-Dawley and Wistar rats," Acta Physiol. Scand. 114, 193-201 (1982).

${ }^{10}$ P. P. Leyssac and L. Baumbach, "An oscillating intratubular pressure response to alterations in Henle loop flow in the rat kidney," Acta Physiol. Scand. 117, 415-419 (1983).

${ }^{11}$ D. J. Marsh, O. V. Sosnovtseva, A. N. Pavlov, K.-P. Yip, and N.-H. Holstein-Rathlou, "Frequency encoding in renal blood flow regulation," Am. J. Physiol. Reg. Integ. Comp. Physiol 288, R1160-R1167 (2005).

${ }^{12}$ D. J. Marsh, O. V. Sosnovtseva, K. Chon, and N.-H. Holstein-Rathlou, "Non-linear interactions in renal blood flow regulation," Am. J. Physiol. Reg. Integ. Comp. Physiol. 288, R1143-R1159 (2005).

${ }^{13}$ K. P. Yip, N.-H. Holstein-Rathlou, and D. J. Marsh, "Mechanisms of temporal variation in single-nephron blood flow in rats," Am. J. Physiol. 264, F427-F434 (1993).

${ }^{14}$ O. V. Sosnovtseva, A. N. Pavlov, E. Mosekilde, and N.-H. HolsteinRathlou, "Bimodal oscillations in nephron autoregulation," Phys. Rev. E 66, 061909 (2002).

${ }^{15}$ O. V. Sosnovtseva, A. N. Pavlov, E. Mosekilde, N.-H. Holstein-Rathlou, and D. J. Marsh, "A double-wavelet approach to study frequency and amplitude modulation in renal autoregulation," Phys. Rev. E 70, 031915 (2004).

${ }^{16}$ K.-P. Yip, D. J. Marsh, and N.-H. Holstein-Rathlou, "Low dimensional chaos in renal blood flow control in genetic and experimental hypertension," Physica D 80, 95-104 (1995).

${ }^{17}$ K. P. Yip, N.-H. Holstein-Rathlou, and D. J. Marsh, "Chaos in blood flow control in genetic and renovascular hypertensive rats," Am. J. Physiol. 261, F400-F408 (1991).

${ }^{18}$ K. P. Yip, N.-H. Holstein-Rathlou, and D. J. Marsh, "Dynamics of TGFinitiated nephron-nephron interactions in normotensive rats and SHR," Am. J. Physiol. 262, F980-F988 (1992).

${ }^{19}$ M. Barfred, E. Mosekilde, and N.-H. Holstein-Rathlou, "Bifurcation analysis of nephron pressure and flow regulation," Chaos 6, 280-287 (1996).

${ }^{20}$ N.-H. Holstein-Rathlou and P. P. Leyssac, "Oscillations in the proximal intratubular pressure, a mathematical model," Am. J. Physiol. 252, F560 F572 (1987).

${ }^{21}$ Y. M. Chen, K.-P. Yip, D. J. Marsh, and N.-H. Holstein-Rathlou, "Magnitude of TGF-initiated nephron-nephron interactions is increased in SHR," Am. J. Physiol. 269, F198-F204 (1995).

${ }^{22}$ A. J. Wagner, N.-H. Holstein-Rathlou, and D. J. Marsh, "Internephron coupling by conducted vasomotor responses in normotensive and spontaneously hypertensive rats," Am. J. Physiol. 272, F372-F379 (1997).

${ }^{23}$ D. Casellas, N. Bouriquet, and L. C. Moore, "Branching patterns and autoregulatory responses of juxtamedullary afferent arterioles," Am. J. Physiol. 272, F416-F421 (1997).

${ }^{24}$ L. C. Nordsletten, S. Blackett, M. D. Bentley, E. L. Ritman, and N. P. Smith, "Structural morphology of renal vasculature," Am. J. Physiol. Heart Circ. 291, H296-H309 (2006).

${ }^{25}$ D. E. Postnov, O. V. Sosnovtseva, and E. Mosekilde, "Oscillator clustering in a resource distribution chain," Chaos 15, 13704 (2005).

${ }^{26}$ B. E. Pitman, R. M. Zaritski, Kesseler, K. J, L. C. Moore, and H. E. Layton, "Feedback-mediated dynamics in two coupled nephrons," Bull. Math. Biol. 66, 1463-1492 (2004).

${ }^{27}$ K. S. Jensen, E. Mosekilde, and N.-H. Holstein-Rathlou, "Self-sustained oscillations and chaotic behavior in kidney pressure regulation," Mondes Development 54/55, 91-109 (1986).

${ }^{28}$ L. C. Moore, A. Rich, and D. Casellas, "Ascending myogenic autoregulation, interactions between tubuloglomerular feedback and myogenic mechanisms," Bull. Math. Biol. 56, 391-410 (1992).

${ }^{29}$ S. Newhouse, D. Ruelle, and F. Takens, "Occurrence of strange Axiom A attractors near quasiperiodic flows on $T^{m}$," Commun. Math. Phys. 64, 35-40 (1978).

${ }^{30}$ V. I. Arnol'd, R. V. Gamkrelidze, N. D. Kazarinoff, V. S. Aframjovich, Y. S. Il'yashenko, and L. P. Shil'nikov, Dynamical Systems (Springer Verlag, New York, 1994), Vol. 5.

${ }^{31}$ C. Grebogi, E. Ott, S. Pelikan, and J. A. Yorke, "Strange attractors that are not chaotic," Physica D 13, 261-268 (1984). 